\title{
Phase-lag synchronization analysis in complex systems with directed inter-relations
}

\author{
V.S.G. Martins ${ }^{1,2}$, A.C. Rodrigues ${ }^{1,3}$, H.A. Cerdeira ${ }^{4}$, and B.S. Machado ${ }^{1, a}$ \\ 1 Hospital Israelita Albert Einstein, São Paulo, Brazil \\ 2 Universidade Federal de Campina Grande, Campina Grande, Brazil \\ 3 Bioinformatics Interunits Graduate Program, University of São Paulo, São Paulo, Brazil \\ 4 Instituto de Física Teórica, Universidade Estadual Paulista, São Paulo, Brazil
}

Received 1 October 2015 / Received in final form 11 January 2016

Published online 29 February 2016

\begin{abstract}
In this work, we proposed a novel way to estimate phase-lag synchronization in coupled systems. This approach was applied into two systems: a directed-coupled Rössler-Lorenz system and a network of Izhikevich neurons. For the former case, the phase-lag synchronization revealed an increase in complexity for the Lorenz subsystem components, when the coupling is activated. The opposite behavior was observed when the Izhikevich network were organized in a hierarchical way. Our results point out to emergent synchronism related to causal interactions in coupled complex systems.
\end{abstract}

\section{Introduction}

The concept of phase synchronization as proposed in 1996 by Rosenblum et al. [1,2], has been widely used and proved experimentally when applied to time series [3-5]. For coupled systems, phase synchronization has been extensively applied in the context of chemical [6] and biological [7-9] systems.

In brain connectivity analysis, the phase synchronization phenomena can be considered a fundamental neural mechanism [10-13], responsible for supporting neural communication and neural plasticity and is probably relevant to many cognitive processes [14-19]. In networks of neurons, the phase determines the degree of excitability of the neurons and influences the discharge times of cells [13]. Consequently, phase relationships between brain regions affect the relative timing of action potentials in those regions [20-22]. Moreover, studies using synchronization to analyse functional and effective connectivity [23-25], applied to neurophysiological data such as fMRI $[26,27]$ and EEG $[28,29]$ allowed a more accurate identification of specific anatomical areas activated during cognitive tasks and other brain states.

In this work, we propose a measure of phase-lag synchronization to analyze coupled systems. This measure is defined by the phase locking value (PLV) [10] between two phase time courses (time delayed by a lag parameter). We apply this approach to simulations of two coupled systems: the Rössler-Lorenz directed coupling and

\footnotetext{
${ }^{a}$ e-mail: birasm@gmail.com
} 
networks of Izhikevich neurons. Our results point out that different coupling mechanisms, although with opposite complexity variation, are followed by the emergence of a synchronized structure. Our analysis shows that increasing the coupling leads to an increase of delayed synchronization structure of the system receiving the interaction (response system). The particular features will be discussed in Sect. 3. This may help to understand how causality and synchronization are related in these systems.

This paper is organized as follows: in Sect. 2 we present the method to study lag synchronization as well as the models under study: coupled Rössler-Lorenz and coupled Izhikevich neuron system. In Sect. 3, we discuss the results. In Sect. 4 we conclude with a summary of the work.

\section{Materials and methods}

\subsection{Phase-lag synchronization}

The concept of phase synchronization was discussed by Rosenblum et al. [1] and has been widely used ever since. Consider the rigorous definition in which the phase difference holds for $m=n=1$ in $\left|\Delta \phi_{t}\right|=\left|m \phi_{i, t}-n \phi_{j, t}\right|$. In order to compute the phase synchronization, it is necessary to know the instantaneous phase of both signals involved. Here, we propose a mean phase weight-averaged energy over all wavelet scales [29],

$$
\phi_{\tau}=\frac{1}{\Gamma_{\tau}} \sum_{\ell}\left|W_{\tau}(\ell)\right|^{2} \phi_{\tau}(\ell),
$$

where $\phi_{\tau}(\ell)=\arctan \Im\left[W_{\tau}(\ell)\right] / \Re\left[W_{\tau}(\ell)\right], \Gamma_{\tau}=\sum_{\ell}\left|W_{\tau}(\ell)\right|^{2}$ and

$$
W_{\tau}(\ell)=\int_{-\infty}^{\infty} f(t) \psi_{\tau}^{*}(\ell, t) d t
$$

is the wavelet transform of a given function $f(t)$ localized at $\tau$ with scale $\ell$. The kernel function used here is defined as $\psi_{\tau}(\ell, t)=\pi^{-1 / 4} \exp (-6 \mathrm{it}) \exp \left(\frac{-\mathrm{t}^{2}}{2}\right)$.

Afterwards, we can calculate the PLV, which characterizes the stability of the phase difference between two time series [27]. The PLV measure is given by

$$
\operatorname{PLV}_{i j}=\frac{1}{T}\left|\sum_{\tau=1}^{T} \exp \left(\mathrm{i} \Delta \phi_{\tau}\right)\right|
$$

where $T$ is the time series length. In perfect phase locking, the PLV equals 1, meaning complete synchronization. In this context, a phase-lag synchronization analysis can be performed considering a lag delay time $d$. Thus, we obtain

$$
\operatorname{PLV}_{i j}(d)=\frac{1}{T}\left|\sum_{\tau=1}^{T} \exp \left(\mathrm{i} \Delta \phi_{\tau}(d)\right)\right|
$$

where $\Delta \phi_{\tau}(d)=\phi_{i, \tau}-\phi_{j, \tau-d}$ and $\phi_{j, \tau-d}$ is the phase-time series estimated from the delayed time series. When $i=j$, a zero-lag phase synchronization has the highest PLV, while a nonzero-lag phase varies from 0 to 1 according with the signal periodic structure. 


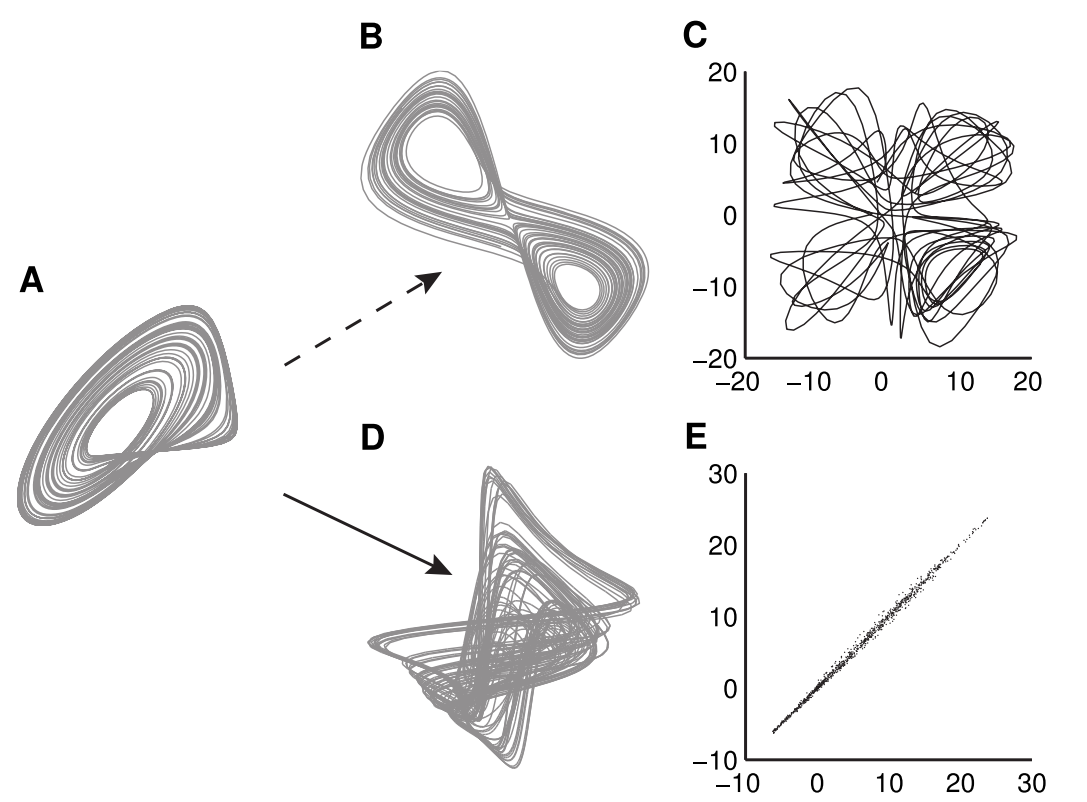

Fig. 1. (A) State-space reconstructions of chaotic system drive Rössler and (B) for the chaotic system response Lorenz, numerically evolved with coupling parameter $\beta=0$. (C) Projection on the plane $\left(y_{1}, \tilde{y}_{1}\right) ; y_{1}(t)$ and $\tilde{y}_{1}(t)$ are two series with different initial conditions $y_{1}(0) \neq \tilde{y}_{1}(0)$. (D) The Lorenz attractor after coupling with the drive system Rössler and (E) the respective $\left(y_{1}, \tilde{y}_{1}\right)$-projection. The diagonal $y_{1}(t)=\tilde{y}_{1}(t)$ indicates that the drive-response system is functionally related.

\subsection{Coupled Rössler-Lorenz system}

Consider the drive-response system, as described by Le Quyen et al. [30]. The system is defined by the directed coupling from a Rössler system

$$
\begin{aligned}
& \dot{x}_{1}=-6\left(x_{2}+x_{3}\right) \\
& \dot{x}_{2}=6\left(x_{1}+0.2 x_{2}\right), \\
& \dot{x}_{3}=6\left(0.2+x_{3}\left(x_{1}-5.7\right)\right)
\end{aligned}
$$

onto a Lorenz system

$$
\begin{aligned}
& \dot{y}_{1}=10\left(y_{2}-y_{1}\right) \\
& \dot{y}_{2}=28 y_{1}-y_{2}-y_{1} y_{3}+\beta x_{2}^{2}, \\
& \dot{y}_{3}=y_{1} y_{2}-8 / 3 y_{3}
\end{aligned}
$$

where $\beta$ is the coupling parameter. The perturbation was applied only to the second equation of the Lorenz system and it does not contain any feedback term. In Fig. 1 we show the attractor reconstruction of the Lorenz system for $\beta=0$ (Fig. 1B) and coupled (Fig. 1D) and the respectives projection onto the $\left(y_{1}, \tilde{y}_{1}\right)$-plane (Figs. 1C and $\mathrm{E}$ ). The $y_{1}$ solution has different initial conditions from those used to $\tilde{y}_{1}$ in numerical simulation. We can observe that the driving system distorts the trajectory of the Lorenz attractor.

To characterize the causality relation between the drive and response systems we employed the method proposed in [30]. Considering the state-space reconstructions 


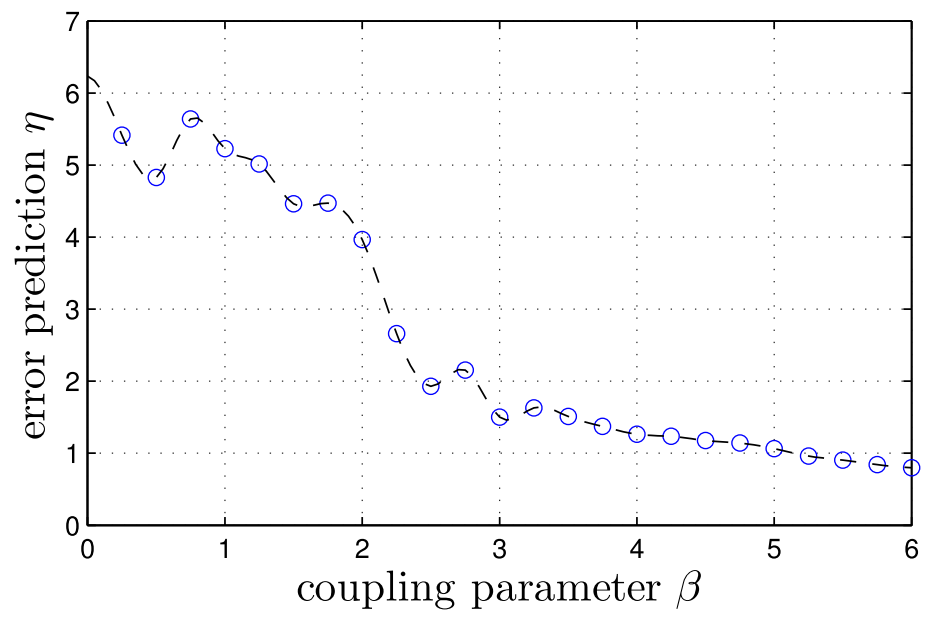

Fig. 2. Error prediction measure $\eta$ as a function of $\beta$. The prediction method was performed considering the causal dependence $x \rightarrow y$.

$\mathbf{x}(t)$ and $\mathbf{y}(t)$, the cross-prediction of $y$ given $x$ is

$$
\hat{y}(t+1)=\frac{1}{\left|U_{\varepsilon}(\mathbf{x}(t))\right|_{\#}} \sum_{\tau / \mathbf{x}(\tau) \in U_{\varepsilon}(\mathbf{x}(t))} y(\tau+1),
$$

where $U_{\varepsilon}(\mathbf{x}(t))=\{\mathbf{x}(\tau):\|\mathbf{x}(t)-\mathbf{x}(\tau)\|<\epsilon\}$ is the neighborhood of $\mathbf{x}(t)$ of radius $\varepsilon$ and $\left|U_{\varepsilon}(\mathbf{x}(t))\right|_{\#}$ is the number of elements in that neighborhood. In this way the cross-predictability can be defined as

$$
\eta=\sqrt{\frac{\sum_{t=2}^{T}(\hat{y}(t)-y(t))^{2}}{T}} .
$$

In Fig. 2 we show the causal dependence as a function of the coupling parameter. It is possible to observe that for $\beta \geq 3$ the one-directional causality relation between the Rössler and Lorenz systems is well established.

\subsection{Izhikevich neurons}

In the second simulation, we created networks using Izhikevich neurons which is a class of pulse coupled neurons, first described in [31]. Four networks were generated with 1000 neurons each, initializing the parameters in the same way as presented in [31] and following the proportion of 4:1 for excitatory-inhibitory neurons. The simulations were integrated using the Euler method with a step of $0.05 \mathrm{~ms}$. The pulses of the neurons from each network were averaged, resulting in a signal similar to a local field potential (LFP). Then, they were analysed using the lag-PLV measure presented here.

The four networks, $z_{1}, z_{2}, z_{3}$ and $z_{4}$, were connected as follows: $z_{i} \rightarrow z_{j}$ for each $j>i$ pair, as shown in Fig. 3. For example, $z_{1}$ influences all other networks but is not caused by them. 


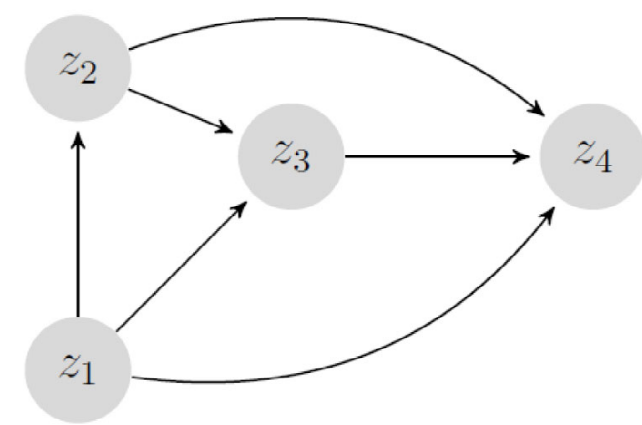

Fig. 3. Connectivity diagram of the Izhikevich's neuron networks.

\section{Results and discussion}

Besides the PLV measure proposed here, we applied the Lempel-Ziv complexity (LZC) $[32,33]$ to characterize the level of complexity of the signals generated under coupling. This measure is normalized between 0 to 1 ; where 0 is for periodic systems, increases for chaotic systems and reaches 1 for random processes. For more details see Kaspar and Schuster [34].

\subsection{The coupling between the Rössler-Lorenz system leads to a phase-lag syncronization in the Lorenz subsystem}

Considering the solutions $x_{1}, x_{2}$ (from Eq. (6)), $y_{1}, y_{2}$ (from Eq. (7)), we calculated the $\operatorname{PLV}(d)$ for two conditions: $\beta=0$ (uncoupling condition) and $\beta=5$ (coupled case). We evaluated this analysis until the lag $d=3$ and we performed a random permutation on the reference signals (with zero lag) in order to get a significance level, i.e., PLV values near or below this threshold are not significant.

For $\beta=0$ we found a high baseline and periodic synchronization signature in the Rössler system - $\left(x_{1}, x_{1}\right),\left(x_{1}, x_{2}\right),\left(x_{2}, x_{1}\right),\left(x_{2}, x_{2}\right)$ pairs - whereas the Lorenz components show a synchronization peak only in zero-lag, declining rapidly to non sinificant levels - $\left(y_{1}, y_{1}\right),\left(y_{1}, y_{2}\right),\left(y_{2}, y_{1}\right),\left(y_{2}, y_{2}\right)$ pairs. This can be seen in Fig. 4 . Moreover, a phase synchronization relation between the Rössler and Lorenz components were not observed - the $(x, y)$-pairs.

For the coupled condition $(\beta=5)$, we observed in Fig. 5 the same synchronization patterns, except for $\left(y_{1}, y_{1}\right),\left(y_{1}, y_{2}\right),\left(y_{2}, y_{1}\right),\left(y_{2}, y_{2}\right)$ pairs where the emergence of synchronization occurs, highlighting an increase of complexity in the Lorenz system components. The LZC increases from $0.042 \rightarrow 0.118$ for $x_{1}$ component and $0.063 \rightarrow$ 0.122 for $x_{2}$ component. Also, a periodic synchronization behavior is evident inside a concave-like curve, indicating another periodic mode.

\subsection{Coupled Izhikevich neurons in a hierarchical way show different synchronization structures for each subnetwork}

Using the LFP from the Izhikevich system described in Sect. 2.3, we analyzed the phase-lag synchronization signature for each subnetwork $\left(z_{1}, z_{2}, z_{3}, z_{4}\right)$ when these networks were under the effect of causal inter-relations. In order to perform this comparison, we simulated the same networks but without interconnections, i.e. they are independent. 

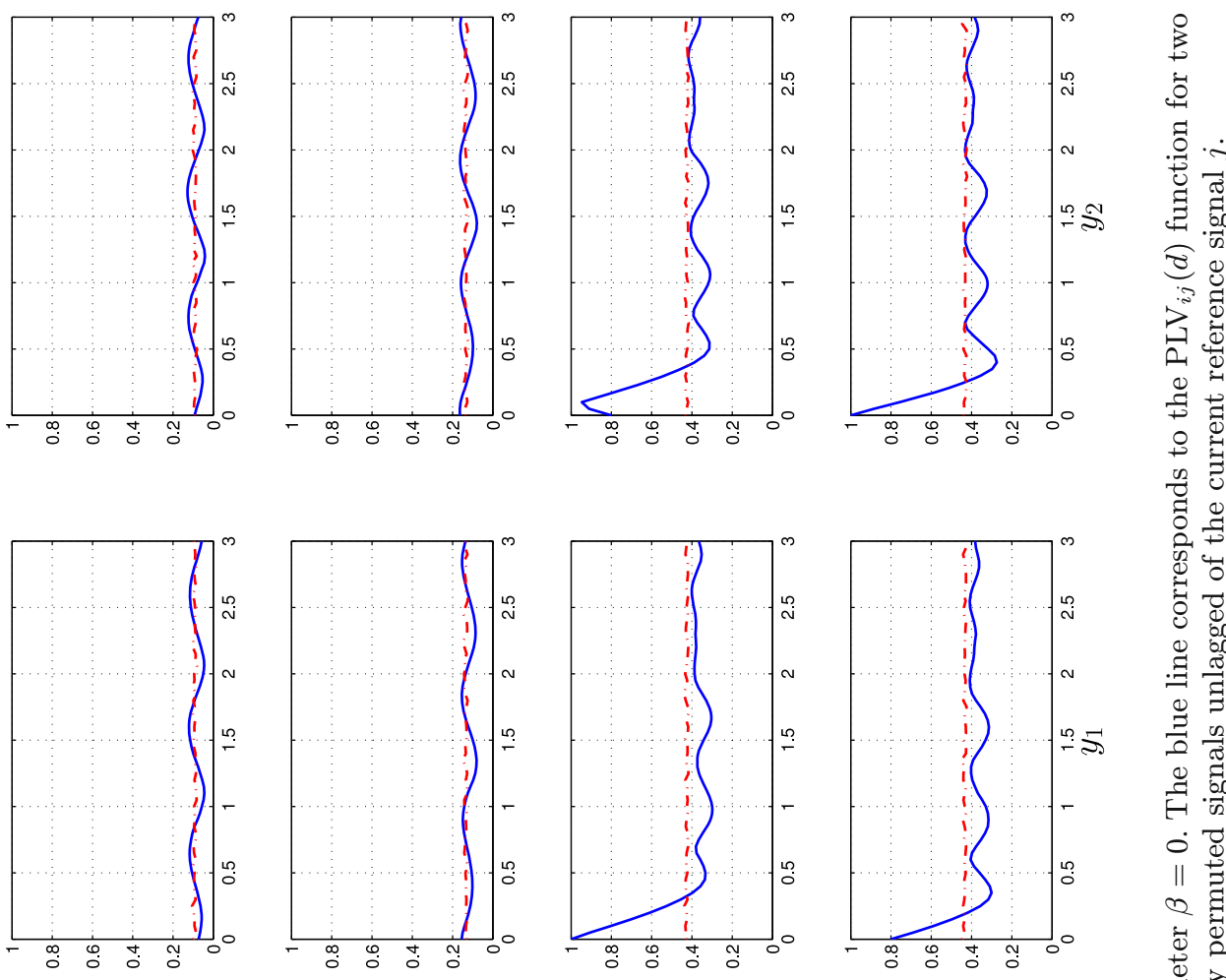

,

$\sum_{=1}^{\infty}$

¿

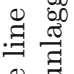

궁 $\frac{\infty}{\pi}$

击 $\cdot \frac{\infty}{6}$

$\circ$

Q

$\dot{0} \stackrel{0}{0}$

हี
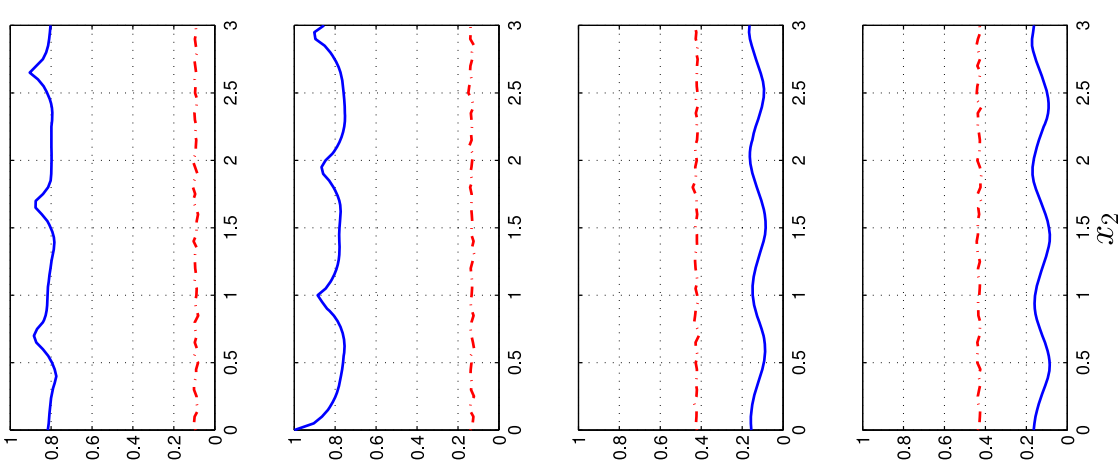

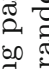

变

苛

造

$\because 0$

永

द्व ठํ

范:

귱 ర్

인
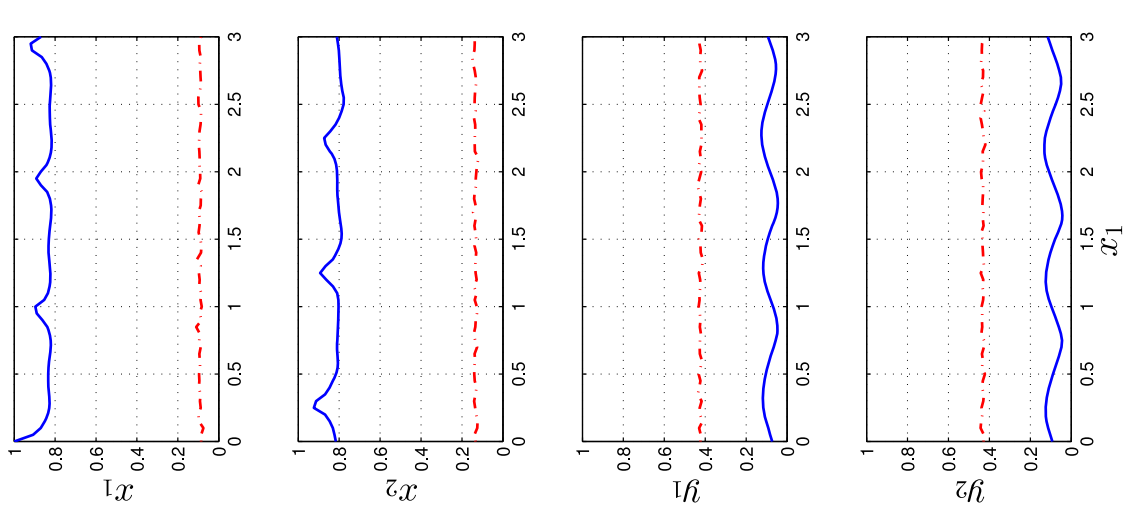

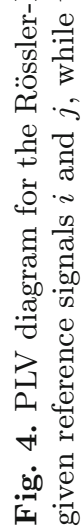



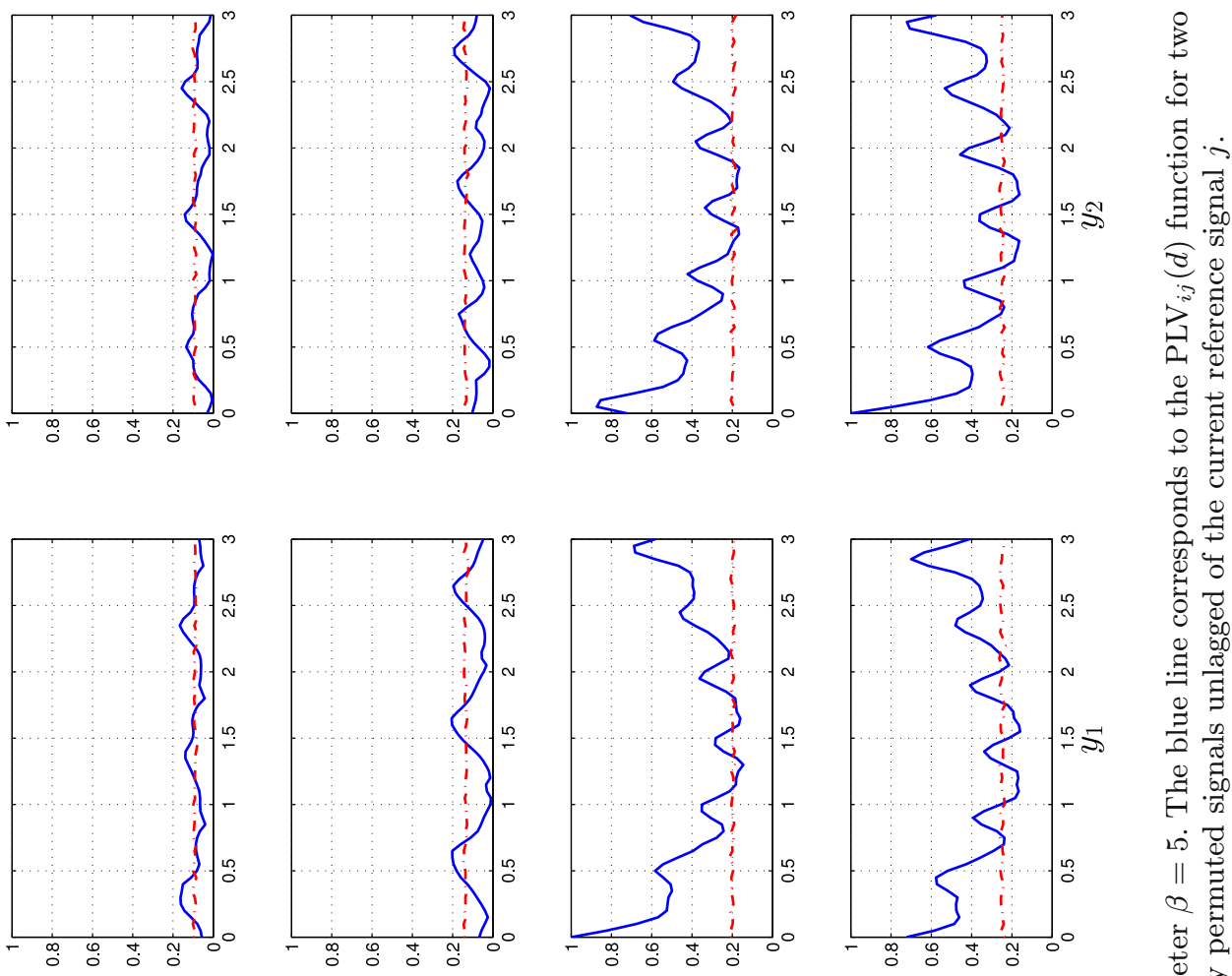

品

in

ठำ

త

을

를

10.

凤

मे

్ㅡ 로
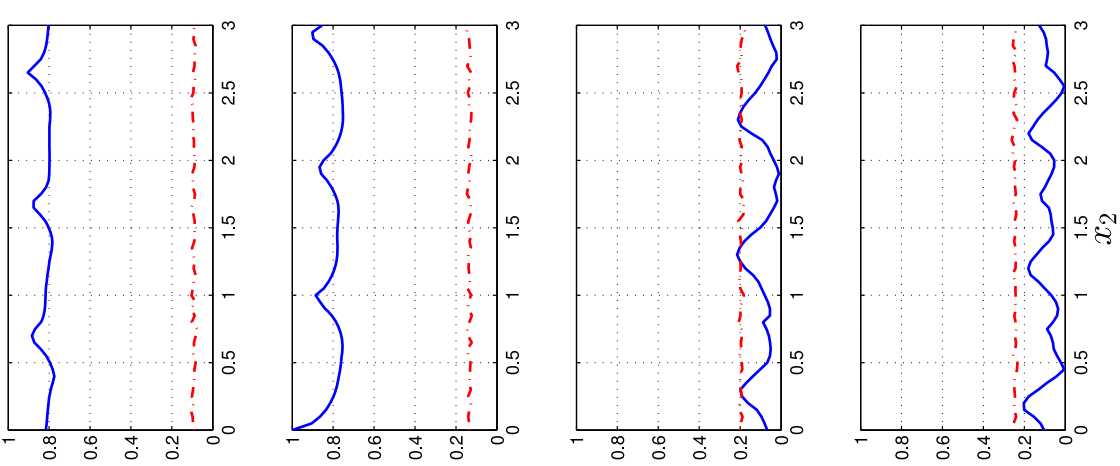

20

变

웅용

赵

.

党

당 ठ

离

궁 ఫ

눙
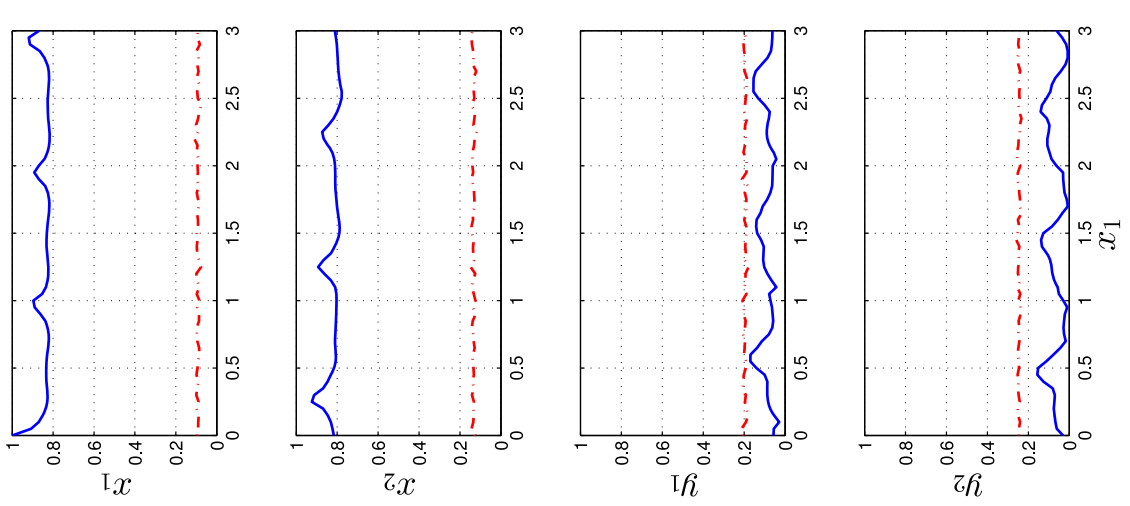

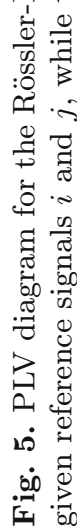


A

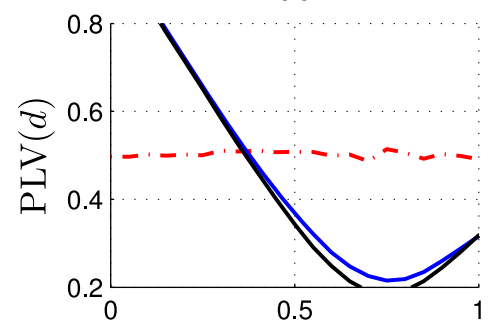

C

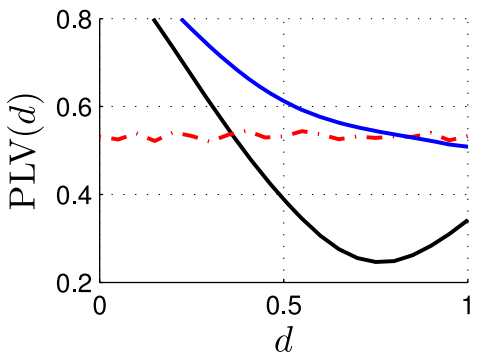

B

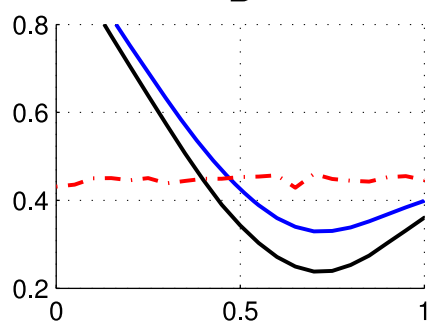

D

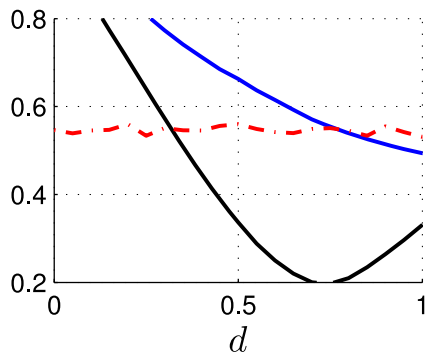

Fig. 6. (A) $\operatorname{PLV}(d)$ for the pairwise $\left(z_{1}, z_{1}\right)$, (B) for $z_{2}$, (C) for $z_{3}$, and (D) for $z_{4}$. The blue line is the PLV $(d)$ considering the ocurrence of coupling among subnetworks and the black line is when the subnetwork are disconnected.

Figure 6 shows the diagonal elements of the $\operatorname{PLV}_{i j}(d)$ diagram. The blue lines are the $\operatorname{PLV}_{i i}(d)$ for each LFP when the subnetworks were connected, while the black line is the $\operatorname{PLV}_{i i}(d)$ when the subnetworks were independent. We can observe an increase in the divergence between these significant curves as the number of interconnections received also increase. For example, in Fig. 6A, it corresponds to $\operatorname{PLV}_{11}(d)$, a subnetwork which does not receive connections, resulting in a small distance between the two significant curves. In Fig. 6D, that corresponds to the $\operatorname{PLV}_{44}(d)-$ with $z_{4}$ receiving causal connections from $z_{1}, z_{2}$ and $z_{3}-$, it exhibits a wider area.

For this case of coupled Izhikevich neurons, the LZC decreases as a function of the directed coupling. The LZC values for each subnetwork are $\operatorname{LZC}\left(z_{1}\right)=0.609$, $\operatorname{LZC}\left(z_{2}\right)=0.597, \operatorname{LZC}\left(z_{3}\right)=0.523$ and $\operatorname{LZC}\left(z_{4}\right)=0.398$. These results, in contrast to results found for the Rössler-Lorenz system, show a decrease of complexity related with a higher level of synchronization.

\section{Conclusions}

In this paper we analyzed two complex systems that exhibit a similar behavior when coupled: they increase the synchronization structure at the cost of changing its complexity. While in the Rössler-Lorenz system the lag synchronization increases when complexity increases, in the Izhikevich networks the complexity drops. It is important to notice that the emergence of synchronization depends on the level of complexity. The difference between the two systems was the way in which the coupling was implemented, which in turn can enlighten the notion of causality interactions and synchronism. It is also important to note that, in both cases, an emergence of synchronization was observed, even if the subsystems were not synchronized with each other.

These results may bring light to understand some biological phenomena related with synchronization as for example, the epilepsy. It is known that during an epileptic seizure, the focus of the crisis recruit other regions, but little is known about this 
propagation mechanism and its origins. Does hyper synchronization happens due to increased internal synchronization in these regions when coupled to the focus of the illness? In the second example of this paper, the neurons of a subnetwork are more synchronized to each other, even without synchronization between the neurons in the subnetwork that drive the causal relation. Moreover, these results could be useful to study synchronization in complex networks where the nodes, rather than being just a simple oscillator, are composed by another complex system.

\section{References}

1. M.G. Rosenblum, A.S. Pikovsky, J. Kurths, Phys. Rev. Lett. 76, 1804 (1996)

2. A. Pikovsky, M. Rosenblum, J. Kurths, Synchronization: a universal concept in nonlinear science (Cambridge University Press, Cambridge, 2001)

3. P. Tass, M.G. Rosenblum, J. Weule, J. Kurths, A.S. Pikovsky, J. Volkmann, A. Schnitzler, H.J. Freund, Phys. Rev. Lett. 81, 3291 (1998)

4. B. Blasius, A. Huppert, L. Stone, Nature 399, 354 (1999)

5. A. Grinsted, J.C. Moore, S. Jevrejeva, Nonlinear Proc. Geoph. 11, 561 (2004)

6. J.F. Totz, R. Snari, D. Yengi, M.R. Tinsley, H. Engel, K. Showalter, Phys. Rev. E 92, $022819(2015)$

7. P.C. Ivanov, M.G. Rosenblum, C.K. Peng, J. Mietus, S. Havlin, H. Stanley, A.L. Goldberger, Nature 383, 323 (1996)

8. A. Stefanovska, H. Haken, P.V.E. McClintock, M. Hozic, F. Bajrovic, S. Ribaric, Phys. Rev. Lett. 85, 4831 (2000)

9. B. Cazelles, M. Chavez, G.C. de Magny, J.F. Guegan, S. Hales, J. R. Soc. Interface 4, $625(2007)$

10. J.P. Lachaux, E. Rodriguez, J. Martinerie, F.J. Varela, Hum Brain Mapp 8, 194 (1999)

11. F.J. Varela, J.P. Lachaux, E. Rodriguez, J. Martinerie, Nat. Rev. Neurosci. 2, 229 (2001)

12. C.J. Stam, G. Nolte, A. Daffertshofer, Hum Brain Mapp 28, 1178 (2007)

13. J. Fell, N. Axmacher, Nature Rev. Neurosci. 12, 105 (2011)

14. P.R. Roelfsema, A.K. Engel, P. Konig, W. Singer, Nature 385, 157 (1997)

15. E. Rodriguez, N. George, J.P. Lachaux, J. Martinerie, B. Renault, F.J. Varela, Nature 397, 430 (1999)

16. A.K. Engel, P. Fries, W. Singer, Nature Rev. Neurosci. 2, 704 (2001)

17. P. Fries, J.H. Reynolds, A.E. Rorie, R. Desimone, Science 291, 1560 (2001)

18. J.M. Palva, S. Palva, K. Kaila, J. Neurosci. 15, 3962 (2005)

19. O. Jensen, J. Kaiser, J.P. Lachaux, Trends Neurosci. 30, 317 (2007)

20. P. Lakatos, G. Karmos, A. Mehta, I. Ulbert, C. Schroeder, Science 320, 110 (2008)

21. C.E. Schroeder, P. Lakatos, Trends Neurosci. 32, 9 (2009)

22. B. Voytek, M. D’Esposito, N. Crone, R.T. Knight, NeuroImage 64, 416 (2013)

23. K.J. Friston, Hum. Brain Mapp. 2, 56 (1994)

24. K.J. Friston, L. Harrison, W. Penny, NeuroImage 19, 1273 (2003)

25. M. Rubinov, O. Sporns, NeuroImage 52, 1059 (2010)

26. C.J. Stam, Nonlinear brain dynamics (Nova Science Publishers, New York, 2006)

27. M. Chavez, M. Valencia, V. Latora, J. Martinerie, Int. J. Bifurcat Chaos 20, 1677 (2010)

28. M. Le Van Quyen, J. Foucher, J.P. Lachaux, E. Rodriguez, A. Lutz, J. Martinerie, F.J. Varela, J. Neurosci. Meth. 111, 83 (2001)

29. A.C. Rodrigues, B.S. Machado, G. Florence, A.P. Hamad, A.C. Sakamoto, A. Fujita, L.A. Baccalá, E. Amaro Jr., K. Sameshima, Eur. Phys. J. Special Topics 223, 2933 (2014)

30. M. Le Van Quyen, J. Martinerie, C. Adam, F.J. Varela, Physica D 127, 250 (1999)

31. E.M. Izhikevich, IEEE Trans. Neural Netw. 14, 1569 (2003)

32. A. Lempel, J. Ziv, IEEE Trans. Inform. Theory 22, 75 (1976)

33. J. Ziv, A. Lempel, IEEE Trans. Inform. Theory 23, 337 (1977)

34. F. Kaspar, H.G. Schuster, Phys. Rev. A 36, 842 (1987) 